\title{
DE LA DOUCEUR MISE EN DISCOURS : PERCEPTION ET ENONCIATION DANS CAHier de Verdure de Philippe JaccotTet
}

Georges HANI

Université de Damiette

\begin{abstract}
En): In Philippe Jaccottet's Cahier de Verdure, we find and test softness a number of times: a quasi-pregnant effect of speech in the seven poems that make up the collection, but we note that this discursive effect which is linked to visual, tactile and auditory perception is considered as a profound language that controls the meaningful process in the collection. Assuming that perception is a semiotic process that involves tensions which dominate discourse and, relying on the data of tensive semiotics, we try to question the sources of softness through an analysis of the poems in the collection.
\end{abstract}

Keywords (En): softness ; tensivity ; semiotics ; perception ; enunciation

Mots-clés (Fr) : douceur ; tensivité ; sémiotique ; perception ; énonciation

\section{Introduction}

Philippe Jaccottet est connu comme le poète de la douceur : à la lecture de ses paysages, de ses souvenirs, on serait tenté d'éprouver « une douceur sans limites » (JACCOTTET, 2012 : 16). Cette douceur n'est pas simplement une ambiance mais plutôt un effet sémantique, à base perceptive dans la mesure où elle appelle nécessairement le corps incarné et désincarné à la fois (DUFOURMANTELLE, 2013 : 18), ressenti à même la lecture du texte poétique : le moi percevant perçoit d'abord la douceur dans la nature puis il transmet cette expérience sensible en discours. Ainsi nous ne sommes pas loin de la notion du passage de l'expérientiel vers le discursif en vertu de laquelle un nombre important de travaux ont abordé la poésie jaccottéenne (Cf. JOSSUA, 2002 : 45 ; SOURDILLON, 1996 : 35, 36 ; SAMSON, 2004 : 85 ; CHALARD, 2005 : 140, 141 ; VIART, 2003 : 23, pour ne citer que quelques exemples). Le poète tient alors à restituer dans et par les mots la synthèse de ce qui s'est offert aux sens, d'autant plus que l'une des opérations suivie par Jaccottet s'avère le creusement du réel (SAMSON, 2004 : 40) en vue de mettre l'objet perçu, dans la plénitude de sa présence, sous les yeux du lecteur (LEMAIRE, 2014 : 75 ; GUERMÈS, 2012 : 103). Écrire, chez Jaccottet, c'est se laisser traverser par la lumière qui franchit les mots tout en les effaçant : une fois la lumière captée, le poète peut ainsi la transmettre en discours : le poète, c'est vraiment l'instrument de ce passage.

Or, dans Cahier de Verdure (2012), la douceur se place non seulement sous le signe du passage mais elle semble une catégorie plutôt figurale qui réside au soubassement du discours et qui se nourrit des tensions profondes comme celles qui se trouvent entre le visible et l'invisible, le limité et l'illimité, le fermé et l'ouvert, l'apparence des choses et ce qui existe réellement (DUFOURMANTELLE, 2013 : 30). Notre but est alors d'explorer l'univers signifiant de la douceur tout en interrogeant ses sources et les tensions qu'elle implique : nous tenterons, plus précisément, de répondre aux questions suivantes : comment la douceur est-elle sémiotisée dans le 
recueil ? De quelle manière la douceur ressentie contrôle-t-elle l'énonciation? Qu'est-ce qui caractérise cette expérience sensible, une fois mise en discours ? Pour ce faire, nous recourons à la sémiotique tensive, élaborée, entre autres, par Claude Zilberberg. Cette approche, se réclamant de la phénoménologie, conçoit le discours comme un espace tensif ou un champ de présence qui s'appuie sur la corrélation entre l'intensité et l'extensité.

\section{Perception et tensivité}

L'approche tensive part du postulat que le processus signifiant découle nécessairement d'un acte perceptif, déjà en soi informé de sens (BEIVIDAS, 2012 : 708). L'expérience perceptive se présente souvent ainsi sous le signe d'une corrélation inverse ou converse entre l'intensité, à savoir l'énergie ou la force et l'extensité, à savoir l'étendue spatio-temporelle (ZILBERBERG, 2008: 100). Le discours fonctionne ainsi comme un espace tensif d'accueil pour les grandeurs accédant dans ce champ de présence : celles-ci se trouvent qualifiées au titre de l'intensité et de l'extensité, l'intensité étant descriptible en subvalences de tempo et de tonicité, l'extensité en temporalité et en spatialité (FONTANILLE, ZILBERBERG, 1998, : 95-96 ; ZILBERBERG, 2012 : 26).

L'intensité et l'extensité sont envisagées comme des catégories perceptives et sémantiques à la fois non seulement parce que tout processus signifiant présuppose un acte perceptif mais aussi parce que l'acte perceptif est en soi sémiotique : le sujet perçoit le monde en vertu d'une sémiose convenue, d'un pacte sémiologique (BEIVIDAS, 2012 : 715) qui tient à transformer les données brutes appréhendées par les sens en une saisie significative : on perçoit seulement ce que notre langage nous conduit à catégoriser (BORDRON, 2007). Notons que le point de départ de cette approche tensive s'avère les lexèmes mis en discours car à travers les sèmes qui $\mathrm{y}$ sont actualisés, on pourrait explorer les subvalences tensives en concordance avec le topos développé par le discours (ZILBERBERG, 2011 : 46). D'ailleurs, Jaccottet travaille patiemment sur les mots afin de diminuer leur opacité (LEMAIRE, 2014 : 79).

\section{Devenir de la douceur}

\section{1. L'intensité diffractée}

La douceur est sémantiquement vue comme le caractère de ce qui n'est pas brusque, qui manifeste de la docilité et qui n'est point sévère (LAROUSSE, 1987 : 400). En termes tensifs, disons qu'elle se caractérise généralement par une tonicité faible (manque de sévérité) et par un tempo lent (absence de brusquerie) : elle relève donc de l'univers signifiant du parvenir et non du survenir, ce dernier impliquant la soudaineté de l'événement qui se saisit du sujet percevant (ZILBERBERG, 2012 : 32). Or, ce que l'on remarque dans Cahier de Verdure est que la douceur se trouve plutôt liée à l'extensité spatio-temporelle qu'à l'intensité affaiblie: le plus souvent l'intensité (avec ses deux versants de tonicité et de tempo) ne se montre que sur le fond d'une extensité bien diffuse. 
Dès les premières lignes du «Cerisier », le poète détermine, comme par avance, la raison d'être de son programme énonciatif : pourquoi écrit-il ? Rassembler les fragments d'une joie qui a déjà explosé, il y a longtemps et dont il ne reste que l'éclat ou plutôt le reflet de l'éclat que le poète tente de retrouver dans la nature (JACCOTTET, 2012 : 11). L'explosion de la joie manifeste une tonicité vive et un tempo rapide: exploser, c'est éclater violemment ou se manifester soudain (LAROUSSE, 1987 : 489) : on a donc affaire aux sèmes de /force/ et de /soudaineté/ qui expriment respectivement la tonicité et le tempo. Mais cette intensité se trouve virtualisée par la grande extensité temporelle (la joie a explosé il y a longtemps) et spatiale parce que cette joie finit par «répand[re] sa poussière en nous» (JACCOTTET, 2012 : 11) et par se fragmenter dans la nature : répandre est défini comme envoyer hors de soi (LAROUSSE, 1987 : 1018) tandis que fragments (le reflet de cet éclat fragmenté dans la nature) s'appliquent aux parties isolées ou rares d'un certain objet (LAROUSSE, 1987 : 536) ; les deux lexèmes contiennent alors une dimension spatiale. Quant à reflet, il se définit comme la réflexion affaiblie de la lumière (LAROUSSE,1987 : 1002) : le sème /faible/ nous renvoie immédiatement à l'idée de la tonicité affaiblie. Nous pourrions ainsi remarquer qu'en ce début de poème, la douceur provient d'une intensité faible et surtout d'une extensité plus ou moins diffuse ou diffractée.

Le poète se rappelle ensuite l'apparition du cerisier : «[...] c'était le soir, assez tard même, longtemps après le coucher du soleil, à cette heure où la lumière se prolonge au-delà de ce qu'on espérait, avant que l'obscurité ne l'emporte définitivement [...]»(JACCOTTET, 2012: 13); l'extensité temporelle se laisse aisément remarquer : prolonger, c'est augmenter la durée (LAROUSSE, 1987 : 947). Ce rayon de «lumière survivante » (JACCOTTET, 2012 : 13), à foyer non identifié, non visible «semble émaner de l'intérieur des choses et monter du sol» (JACCOTTET, 2012 : 13), du chemin de terre ou du champ de blé. La tonicité lumineuse est ainsi très faible et le tempo est sensiblement lent mais l'extensité spatiale de la lumière s'avère diffuse car la source en est encore non identifiée. Rappelons que le verbe émaner a le sème /lent/ puisqu'il se définit comme provenir, se dégager (LAROUSSE, $1987: 427$ ).

Même quand une métamorphose s'est produite suite à l'apparition du cerisier, la tonicité est vite remise en cause par l'étendue spatiale presque illimitée du changement : la métamorphose qui est un changement de forme ou de structure survenant chez un être ou dans une chose (LAROUSSE, 1987 : 745) semble cumuler les sèmes de /soudaineté/ et de /force/ mais cette intensité se dilue vite en extensité car le sol est devenu «de la lumière » (JACCOTTET, 2012 : 13), le blé « évoquait l'acier » (JACCOTTET, 2012 : 13), «les contraires se rapprochaient, se fondaient » (JACCOTTET, 2012 : 13) et plus loin «l'herbe et la faux ne faisaient plus qu'un » (JACCOTTET, 2012:15). Rappelons qu'on passe ainsi spatialement du rapprochement à la fusion, puis de celle-ci à l'identité totale. Résultat : nous (je + on) nous trouvions reconduits temporellement «très loin en arrière dans le temps » (JACCOTTET, 2012 : 14), «vers cet âge imaginaire » (JАCCOTTET, 2012 : 14) et spatialement « tout au fond de nous-mêmes » (JACCOTTET, 2012 : 14). Cette étendue spatio-temporelle se faisait d'une manière « presque imperceptible et tendre comme une caresse » (JACCOTTET, 2012: 14) : la caresse porte le sème /faible /car elle 
concerne l'effleurement de la main (LAROUSSE, 1987 : 192) mais cette intensité affaiblie se place sous le signe de l'étendue.

Cependant, cette extensité spatio-temporelle aboutit paradoxalement dans les lignes qui suivent à une restriction spatiale puisque «le monde offrait les apparences rassurantes d'une maison ou même quelquefois d'un temple» (JACCOTTET, 2012 : 14) : on passe ainsi de l'espace ouvert et non limité à l'espace fermé d'une maison ou d'un temple, ou en d'autres termes, de l'extensité spatiale diffuse à l'extensité plus ou moins concentrée. Mais il ne s'agit en effet que d'apparences qui se définissent comme étant un aspect extérieur qui répond plus ou moins à la réalité (LAROUSSE, 1987 : 63). L'univers est assimilé à une maison où nous marchions et qu' « une lampe invisible éclairait sourdement » (JACCOTTET, 2012 : 14) : la tonicité est très faible puisque sourdement veut dire peu sonore, dont le bruit est étouffé (LAROUSSE, 1987 : 1543) et le tempo est bien lent : éclairer à l'imparfait dénote la longue durée; la douceur semble ici encore liée tant à l'intensité affaiblie (tonicité faible et tempo lent) qu'à l'extensité spatiale concentrée. Mais vite, on revient à la diffusion spatiale car « le ciel était comme une paroi de verre vibrant à peine au passage de l'air rafraîchi » (JACCOTTET, 2012 : 14). La faiblesse de la tonicité provient ici de la quasi absence de mouvement: vibrant à peine. Quant à la dimension temporelle, on est encore dans le cadre de l'étendue diffuse car c'est bien la vie qui offre les apparences d'une musique: rappelons que la vie signifie l'existence humaine envisagée dans sa durée totale de la naissance à la mort (LAROUSSE, 1987 :1231).

De cette apparition en souvenir du cerisier, il ne reste que «le reflet très affaibli [...] qui (lui) parvenait encore » (JACCOTTET, 2012 : 14) ; l'accent est ainsi mis sur la faiblesse de la tonicité car à côté du reflet qui est une réflexion affaiblie de la lumière, on trouve l'adjectif affaibli modalisé par très: la faiblesse est donc dédoublée et le tempo est lent : le verbe parvenir et l'imparfait de durée l'affirment. Le silence s'étend spatialement : «Le silence était moins rompu qu'agrandi par l'aboi d'un chien [...] » (JACCOTTET, 2012 : 14). La faiblesse de la tonicité est ici liée à l'absence de bruit. À cet affaiblissement auditif, fait suite une faiblesse de la tonicité lumineuse : «Un vantail plaqué d'une mince couche d'argent avait tourné vers nous son miroitement » (JACCOTTET, 2012 : 14) ; nous ne sommes pas loin ici de la constitution sémique du reflet qui dénote la faiblesse de la lumière : miroiter, c'est réfléchir la lumière tout en jetant des reflets par intervalles (LAROUSSE, 2012 : 754) donc, tonicité affaiblie et tempo sensiblement lent .

C'est bien sur le fond de cette faiblesse de tonicité auditive, lumineuse et kinésique (le mouvement) et de tempo ralenti qu'apparaît enfin le cerisier chargé de fruits mais cette apparition ne s'est pas faite soudain : c'est alors, c'est là (JACCOTTET, 2012: 14); elle se place plutôt sur la dimension de l'extensité spatiale : relativement loin, de l'autre côté, à la frontière du champ, «parmi d'autres arbres de plus en plus sombres» (JACCOTTET, 2012: 14). Lorsque le poète commence à décrire le cerisier, on constate qu'il le fait sur le mode de l'étendue surtout spatiale : "Ses fruits étaient comme une longue grappe de rouge, une coulée de rouge, dans du vert sombre [...] du rouge dans du vert à l'heure du glissement des choses les unes dans les autres, à l'heure d'une lente et silencieuse apparence de métamorphose [...]» (JACCOTTET, 2012: 15) : la coulée se définit comme une 
quantité de matière en fusion qui se répand (LAROUSSE, 1987 : 346), glissement signifie se déplacer d'un mouvement continu sur une surface lisse (LAROUSSE, 1987 : 573). L'intensité perceptive est tellement faible que le sujet ne peut pas bien délimiter les contours de l'arbre et de ses fruits et feuilles : une coulée de rouge dans du vert sombre, des fruits dans un berceau. Comme les deux lexèmes coulée et glissement contiennent les sèmes /mouvement/, /lent/ ils relèvent de la spatialité et du tempo ralenti : c'est donc la même corrélation entre une intensité affaiblie et une extensité diffuse.

Ce rouge assimilé au feu qui ne brûlait pas (JACCOTTET, 2012:15), «ne mordait pas », «ne crépitait pas » (JACCOTTET, 2012:16) manifeste sûrement la faiblesse de tonicité. Cependant, il semble que quelqu'un ait semé de tels feux au hasard dans la campagne (JACCOTTET, 2012:15) : la tonicité affaiblie ne s'exprime que liée à une extensité surtout spatiale. La même idée d'une étendue spatiale s'affirme encore puisque ces feux que sont les grappes de cerises ne montent pas comme les flammes mais plutôt ils semblent couler ou pendre "dans l'abri des verdures très sombres » (JACCOTTET, 2012 : 16). Toute la visée perceptive du sujet se concentre sur les grappes de cerises de sorte qu'il ne peut « en détacher les yeux» (JACCOTTET, 2012 : 16) ni entendre «la rivière lointaine » (JACCOTTET, 2012 : 17). Il s'ensuit qu'une douceur extensive «frémissait sur tout cela comme un souffle d'air [...]» (JACCOTTET, 2012:16) : cette douceur s'étend spatialement et se présente, quant à l'intensité, sous le signe de la faiblesse de la tonicité et de la lenteur du tempo: frémir se définit comme être agité d'un léger tremblement (LAROUSSE, 1987 : 544) et l'imparfait ne fait qu'allonger la durée.

Le cerisier serait ensuite assimilé à « un petit monument naturel [...] une sorte de pilier mais capable de frémir, même si à ce moment-là, il paraissait absolument immobile »(JACCOTTET, 2012: 17) : on voit ainsi que la tonicité s'affaiblit jusqu'à l'atonie : l'absence totale du mouvement et du tempo mais l'extensité temporelle ne tarde pas gagner la scène : "Ce soir-là, peut-être que, sans en prendre conscience, je sentais que du temps, des heures pendant lesquelles moi-même j'avais vécu [...] avaient pénétré lentement dans ces fruits [...] » (JACCOTTET, 2012 : 18) : le tempo lent de la pénétration s'accouple avec une extensité temporelle relativement étendue. Et le poète de souligner que les fruits couvés par les feuilles vertes « contenaient en suspens tout cela (JACCOTTET, 2012: 18) et que celles-ci ne tardent pas à devenir plus noires que le ciel au bas duquel «elles frémissaient, dans leur sommeil, à peine... » (JACCOTTET, 2012: 18) : la tonicité est graduellement affaiblie et le tempo se ralentit à l'approche de la nuit (le sommeil) : rien qu'un frémissement pendant le sommeil nocturne.

À cette douceur qui provient de la faiblesse d'intensité fait suite une autre qui provient cette fois de l'extensité spatiale : «Une fête lointaine, sous des arceaux de feuilles. À distance, à toujours plus grande distance » (JACCOTTET, 2012 : 19) : les cerises rouges couvées par les feuilles vertes assombries par la nuit sont représentées sous le signe d'une fête lointaine. L'extensité spatio-temporelle est source inépuisable de douceur dans le poème : vers la fin, le poète constate que «certains lieux, certains moments nous "inclinent" » (JACCOTTET, 2012: 19) et nous invitent ainsi à changer de direction ou de perception. 
Dans Cahier de verdure, l'apparition soudaine de la couleur rose cède la place ensuite à «beaucoup d'eau vaillante dans les rochers et des violettes en plein sentier » (JACCOTTET, 2012 : 23) : le tempo accéléré du début dû à un événement perceptif rappant est remplacé par l'extensité spatiale comme si le rose n'apparaît qu'avec l'eau et les violettes qui s'étendent spatialement. L'extensité spatiale se montre aussi dans «Cette combe verte [...] cette espèce de terrasse verte, au-dessus de laquelle passent les nuages [...] ces pâturages où il n' y a plus de bétail» (JACCOTTET, 2012 : 24) : cette étendue spatiale plus ou moins diffuse sera ensuite liée à une intensité faible, due surtout à la faiblesse de la tonicité lumineuse qui marque la fin du jour : "Dans la lumière brillante qui, à contre-jour, s'embrume, cette sorte de hamac d'herbe [...] la bergerie d'ivoire usé comme une lampe restée allumée en plein jour [...] » (JACCOTTET, 2012 : 24) : à contre-jour, c'est l'éclairage d'un objet recevant la lumière de côté (LAROUSSE, 1987 : 668), s'embrume, c'est manquer de clarté (LAROUSSE, 1987 : 168) : on remarque ainsi que les deux ont en commun le sème /lumière faible/ mais usé (détérioré par le fait de l'emploi constant, LAROUSSE, 1987 : 1207) et la lampe restée allumée dénote la durée, donc l'extensité temporelle étendue. La corrélation que l'on peut ainsi dégager et qui constitue l'un des ressorts de la douceur semble donc la suivante : la tonicité lumineuse affaiblie correspond à une extensité temporelle bien diffuse. L'extensité spatio-temporelle atteint son degré ultime de diffusion à la fin du poème lorsque le printemps devient « poussière lumineuse » (JACCOTTET, 2012: 24) qui semble faire pendant à la poussière de la joie répandue en nous qui ouvre «Le Cerisier ».

Dans «Blason vert et blanc», l'expérience perceptive est explicitement présentée dès le début: un verger de cognassiers aperçu «à travers la portière embuée d'une voiture » (JACCOTTET, 2012: 27). C'est encore la faiblesse de la tonicité lumineuse dont on a déjà fait acte dans «Cahier de verdure ». Le sujet percevant a ensuite concentré toute son attention perceptive sur ces arbres de sorte qu'il finit par «oublie[r] les pommiers, les poiriers de [s]on pays natal» (JACCOTTET, 2012 : 27), il regarde longuement tout en s'attardant à son souvenir : comme ce qui se passe souvent, l'intensité perceptive cède la place à une extensité temporelle puisque l'imparfait (regardais, m'attardais) et le verbe s'attarder dénotent la durée.

Le sujet voit que «cette floraison» (JACCOTTET, 2012 : 28) et quelques lignes plus tard «L'ensemble, fleurs et feuilles [qui] avait quelque chose de plus solide, de plus simple, de plus calme [...]» (JACCOTTET, 2012 : 28) se placent sous le signe de l'atonie : aucun mouvement ne s'y fait sentir : "Cela ne vibrait ni ne frémissait comme oiseaux avant l'envol; cela ne semblait non plus commencer, naître ou sourdre [...] » (JACCOTTET, 2012 : 28). Cependant, aucune impression de fragilité ne se dégage de cette scène : on a donc affaire à une tonicité affaiblie reliée à une temporalité figée comme si le non-mouvement était considéré comme le plan d'expression de cette étendue temporelle sans début, ni fin: "c'était là, simplement. » (JACCOTTET, $2012: 28$ )

À l'approche de ces arbres, le sujet assimile «leurs fleurs blanches, à peine teintées de rose » (JACCOTTET, 2012: 30) à de la cire, à de l'ivoire et à du lait (JACCOTTET, 2012 : 31) et à partir de là on assiste à un processus de restriction spatiale: «Étaient-elles [les fleurs] des sceaux de cire, des médailles d'ivoire 
suspendues dans cette chambre verte, dans cette maison tranquille? ? (JACCOTTET, 2012 : 31), «Elles m'on fait penser aussi aux fleurs de cire que l'on voyait autrefois sous des cloches de verre dans les églises [...]» (JACCOTTET, 2012:31): de l'espace ouvert, on passe à l'espace fermé : presque le même processus de rétrécissement spatial que nous avons rencontré dans «Le Cerisier»: la douceur provient ici de la concentration spatiale qui ne cesse de s'exprimer encore sous d'autres figures : après avoir vu le verger comme une chapelle, un oratoire (le sujet a déjà envisagé le cerisier comme un pilier), le sujet percevant le voit comme «la demeure parfaite aux murs poreux et à la toiture légère » (JACCOTTET, 2012 : 34) où le mélange de la lumière et de l'ombre se fait remarquablement sentir : poreux qui se définit comme le caractère de ce qui laisse passer le liquide, la lumière par ses pores (LAROUSSE, 1987 : 912) nous fait immédiatement penser à la faiblesse de la tonicité lumineuse déjà exprimée sous différentes figures visuelles (dont à contrejour, la portière embuée...) : on est ainsi dans le cadre d'une tonicité affaiblie et d'une spatialité concentrée. La douceur ne provient pas seulement de la tonicité lumineuse très faible mais aussi auditive : on y entend « une musique de chalumeaux et de petits tambours encore assourdis par un reste de brume »(JACCOTTET, 2012 : 34) : mais comment la brume peut-elle assourdir ? Ce ne sera plausible que dans le cadre d'une synesthésie où les sens (ici, la vue et l'ouïe) fonctionnent de concert : la faiblesse de tonicité semble ainsi généralisée aux différentes formes de perceptions. La même idée de la faiblesse auditive se retrouve quelques lignes plus loin, mais accouplée cette fois à un tempo explicitement ralenti : le sujet perçoit les fleurs blanches des cognassiers comme une source de lait dans l'herbe « mais il faut que le pas en ces abords ne soit plus entendu, que l'esprit et le cour ralentissent ou presque s'oublient [...] » (JАCCOTTET, 2012: 35).

Comme la douceur se trouve le plus souvent liée à l'extensité, on trouve que le poète, parlant de la couleur verte, postule que celle-ci accorde «dans ses profondeurs jour et nuit [...] tous herbages, tous feuillages » (JACCOTTET, 2012 : 36) et l'extensité spatiale va croissant jusqu'à embrasser le monde en entier : «Vues dont le mouvement, comme celui des oiseaux, recoudrait l'univers » (JACCOTTET, 2012 : 37) : le verbe recoudre qui signifie réunir, de nouveau, les bords (LAROUSSE, 1987 : 303) ne fait qu'affirmer cette extensité spatiale. Celle-ci s'exprime ensuite sous forme plus dynamique avec le verbe passer et encore avec le mouvement exploratoire des yeux : "On passait. On a bu ce lait de l'ombre, en avril, avec ses yeux» (JACCOTTET, 2012: 37) : le corps propre du sujet percevant parcourt ainsi l'espace qui se présente à ses yeux.

L'extensité spatiale se rencontre dans la dernière partie du poème écrite en italique qui commence par ce paragraphe : «La pluie est revenue, sur les feuillages en quelques jours multipliés, épaissis. On aurait dit qu'une ombre était prisonnière de cette cage fragile. » (JACCOTTET, $2012: 39)$, « [...] en quelques jours, tout n'est plus que grottes, pavillons [...] » (JACCOTTET, 2012 : 39) : ici, l'étendue spatiale (la multiplication des feuillages) va de pair avec un tempo relativement rapide (les feuilles se sont épaissies, multipliés en quelques jours ). Mais, cette extensité est vite remise en cause du fait d'un rétrécissement : les feuillages ; vus de l'intérieur, formeraient une cage où la lumière s'affaiblit certainement. La tonicité lumineuse est ensuite doublement affaiblie : "Comme quand traîne une peu de brume sur une - 109 - 
source qui a pris la couleur des plantes qui/l'abritent, un trouble embue» (JACCOTTET, 2012 : 39, 40) : la brume affaiblit la lumière qui plane sur la source où les plantes se reflètent: il s'agit alors d'un voile «qui amortit et qui aiguise » (JACCOTTET, 2012 : 40) la tonicité des profondeurs venues de la source nous ne sommes pas ainsi loin de l'idée des reflets lumineux déjà exprimés. Rappelons que la brume fonctionne ici, sur le plan perceptif, comme un actant de contrôle dont le rôle est de régler l'intensité perceptive entre la source et la cible (FonTANILLE, 1999). Dans la source, le sujet perçoit tout en extensité : «Des êtres jamais vus » (JACCOTTET, 2012 : 40), le ciel «plus haut» (JACCOTTET, $2012: 40$ ) reflété dans « cette source couleur d'herbe » (JACCOTTET, 2012 : 40).

Dans «Sur les degrés montants », le poète décrit le chant des alouettes à la fin de la nuit et au début du jour dont il ne voit «que le reflet blafard peindre, très lentement, vaguement, les rochers » (JACCOTTET, 2012:43) : à la faiblesse de la tonicité lumineuse exprimée par reflet et redoublée par blafard est associé un tempo lent et une extensité diffuse. Leur vol s'étend ainsi spatialement: le sujet ne les distingue pas mais il entend seulement la voix de leur élévation de plus en plus haut (JACCOTTET, 2012 : 43) : ces alouettes avaient jailli «toutes ensemble ou presque, nombreuses, absolument invisibles [...] comme des fusées sonores; ou plutôt, m'a$t$-il semblé tandis que j'écoutais [...] » (JACCOTTET, 2012 : 43) : il semble ainsi que l'extensité spatiale diffuse semble ici liée surtout à l'affaiblissement de l'énergie perceptive : le poète entend seulement et ne voit presque rien parce que « la nuit était encore totale » (JACCOTTET, 2012: 44): la douceur provient ainsi de l'affaiblissement de l'acuité perceptive. Rappelons que le chant des alouettes est présenté dans le reste du poème sous le signe de la tonicité forte car ces oiseaux chercheront à soulever le ciel assimilé à «une sorte de chapiteau, de dais aussi invisible qu'elles » (JACCOTTET, 2012 : 44), « le couvercle énorme de la / nuit » (JACCOTTET, $2012:$ 44, 45).

Dans la dernière partie du poème, écrite en italique, le poète commence par une extensité diffuse liée à une tonicité très faible : "Montagne à contre-jour dans le matin d'été : c'est simplement de l'eau » (JACCOTTET, 2012 : 48) : la faiblesse de la tonicité lumineuse aboutit à une extensité spatiale : tout est devenu de l'eau. Quelques lignes plus loin : même quand le tonnerre qui roule, ce n' est que «Pendant toute une nuit et sur tout le tour de l'horizon » (JACCOTTET, $2012: 49)$ : donc, une extensité spatio-temporelle bien diffuse. Il s'ensuit que le tonnerre se présente sous le signe de «longues percussions têtues d'un orchestre d'Orient lointain» (JACCOTTET, 2012 : 49) : les percussions nous font tout de suite penser au reflet au plan visuel, donc la tonicité auditive est affaiblie du fait de l'extensité spatiale (lointain).

Dans «Éclats d'août », la douceur se place au niveau du ciel : «Cette nuit, / Un vent glacé fouette les astres ; / On dirait / Qu'eux aussi flambent plus avides» (JACCOTTET, 2012 : 54) et les «nuages [sont] assis en majesté comme des dieux» (JACCOTTET, 2012 : 54) : nous avons ainsi affaire aux astres, aux nuages et au vent d'en haut ; il s'agit donc d'une douceur spatialement extensive dont la scène est bien l'espace très ouvert du ciel. Dans ce ciel à l'aube, la montagne semble prendre «la couleur de la violette» (JАCCOTTET, 2012: 57) : encore l'idée de l'extensité spatiale. Cette douceur va même jusqu'à gagner l'univers en entier : «Toute la 
journée du 26 septembre 1988, le monde sous mes yeux est resté immobile dans la sérénité la plus grande [...] le Ventoux m'a fait l'effet d'un lointain sphinx assis sur notre seuil pour le garder du moindre trouble » (JАCCOTTET, 2012 : 57) : l'idée d'une absence quasi-totale de mouvement, donc tempo nul, accompagné d'une faiblesse remarquable de tonicité (sérénité : le caractère de ce qui est calme, de ce qui est sans bruit ni trouble, LAROUSSE, 1987 : 1087) ne se montre que sur le fond d'une étendue cosmique qui joue avec les grandes distances: le Ventoux qui se dresse très loin, et la lune vue comme «Le corps lointain, caché, proche parfois et moins caché» (JACCOTTET, 2012: 58) en sont des exemples. Notons, dans le dernier exemple, la dialectique proxémique: lointain/proche qui dépend principalement de la perception du sujet.

Parlant des couleurs de l'après-midi, le poète les place d'abord sous le signe de la tonicité faible : «le brun qui tire sur le fauve ( (JACCOTTET, 2012: 58) mais il ne manque pas de faire ensuite allusion aux «lointains bleus » (JACCOTTET, 2012: 58) et au ciel «entre l'horizon et de longs nuages » (JACCOTTET, 2012: 58) qui dénotent l'extensité spatiale bien étendue. Face à cette douceur en extension, les yeux ne peuvent qu'adopter une attitude exploratoire qui «embrass[e] d'un même regard la navigation, là-haut, et tout en bas l'heureuse rumeur du port» (JACCOTTET, 2012 : 59). L'idée du glissement d'une couleur dans une autre (cf. la coulée) revient à la fin du poème : les nuages sont assimilés à des anges «dont le corps rosit dans le jaune des ciels d'hiver. » (JACCOTTET, 2012 : 59) : le jaune de l'après-midi semble affaiblir la tonicité du rouge, tout en le transformant en rose mais cela ne se produit qu'à l'envergure du ciel, donc au niveau de l'extensité spatiale.

Dans «Fragments soulevés par le vent », le poète nous avertit :

«Rappelez-vous :

S'il peut être une foudre lente

Et tendre à en mourir,

Irradiant le corps,

c'est cela dont mourir vous privera. »(JАCCOTTET, 2012 : 64)

La lenteur et la faiblesse de la foudre sont liées à sa capacité d'expansion tout au long du corps : irradier, c'est se propager ou s'étendre à partir d'un centre (LAROUSSE, 1987 : 656). On retrouve la faiblesse de la tonicité lumineuse dans : «Par la porte-fenêtre où d'ordinaire entre le jour, filtré par le feuillage [...]» (JACCOTTET, 2012: 65) : la lumière est affaiblie à cause du filtrage des feuilles et le poète revient immédiatement à l'extensité, cette fois temporelle :

«Dans l'ancien monde,

à presque chaque orage,

répondaient une nymphe dévêtue

et un berger tranquille » (JАССОTТЕT, $2012: 66)$

Bien que le poète commence ces vers avec une concentration temporelle, «En cette nuit / En cet instant de cette nuit [...]» (JACCOTTET, 2012 : 70), on constate que l'extensité spatiale ne tarde pas à gagner la scène énonciative : on a affaire à des 
« dieux [qui] incendiaient / le monde [...]» (JACCOTTET, 2012 : 70). La même extensité spatiale, accouplée à une étendue temporelle, on la rencontre lorsque le poète nous parle des couleurs des soirs d'hiver : «[...] comme si l'on marchait de nouveau dans les jardins de Cordoue » (JACCOTTET, 2012 : 71). Les pâtres auraient continué à chanter seuls dans la montagne, si

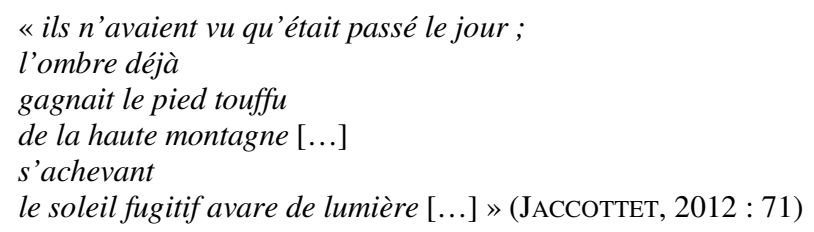

La douceur provient ainsi de la faiblesse de la tonicité lumineuse qui marque le couchant du soleil : le jour est passé, l'ombre commence à s'étendre spatialement et le soleil n'envoie plus ses rayons : on a donc affaire à une tonicité affaiblie et à un tempo ralenti (gagner: envahir progressivement, LAROUSSE, 1987: 555, et l'imparfait dénote la durée) sur fond d'une extensité spatio-temporelle : la montagne au temps du coucher. Rappelons que l'idée du glissement, à tempo lent, de l'ombre dans la lumière nous fait immédiatement penser aux cas similaires du recueil où Jaccottet conçoit le plus souvent l'espace-temps. À cette tonicité lumineuse, on doit ajouter celle d'ordre plutôt auditif: «Les fontaines tintent aux versants les plus hauts de la montagne » (JACCOTTET, 2012 : 72).

La dialectique de la lumière et de l'ombre, ou plutôt de la tonicité lumineuse et de l'extensité spatiale se retrouve dans ce passage :

«Je me souviens de la montée de l'ombre nocturne dans le vallon, comme d'une eau fraîche, cette masse d'obscurité venue de fond du paysage alors que les cimes luisent encore comme des bougies, des cierges, que les lampes des rares maisons de bois s'allument et que les étoiles reparaissent, après que l'éclat du soleil les a fait tout le jour oublier: ces points lumineux épars en haut comme en bas et auxquels on dirait que répondent les sonnailles inégales, intermittentes, dispersées, elles aussi, des troupeaux restés dehors dans l'herbe épaissie des pâtures : comme enfin parle à la lumière lunaire de la neige une longue fontaine haut perdue » (JACCOTTET, 2012 : 72).

On est encore au même moment de glissement de l'ombre dans la lumière : l'obscurité monte progressivement; à tempo ralenti, et sous la forme d'une masse ténébreuse qui s'étend spatialement tout en balayant lentement les points lumineux dispersés en haut et en bas : la faiblesse de la tonicité lumineuse ne se montre que sur le fond d'une extensité spatiale et temporelle. À cette faiblesse lumineuse répond une autre faiblesse auditive : les sonnailles intermittentes mais dispersées, donc qui s'étendent spatialement.

Dans «Apparition de fleurs », le poète situe dès le début le cadre où se déroule l'événement perceptif, à savoir l'apparition de trois fleurs à couleurs distinctes, sous le signe d'une extensité spatio-temporelle « [...] c'était l'été, je passais presque tous les jours le long d'une prairie fleurie» (JACCOTTET, 2012: 77). Bien que l'apparition des fleurs soit présentée sous une forme tensive : certaines seulement en sont visibles le matin à cause de la grande chaleur. Dans la dernière partie du 
poème écrite en italique, le poète décrit la lune assimilée à une serpe «lointaine, mais présente » (JACCOTTET, 2012: 86) : la tension extensive entre le proche et le loin se fait ainsi sentir. La lune émet de la lumière affaiblie qui prend la forme d'un reflet «[...] elle est encore là, une fois de plus, elle m' est donnée sans bruit, sans histoire et pas à moi seulement comme depuis le commencement du monde [...]» (JACCOTTET, 2012: 86) : la faiblesse de la tonicité lumineuse et auditive se trouve ainsi liée à une extensité temporelle (depuis le commencement du monde) et le poète de se rappeler "le cortège d'enfants porteurs de lanternes allumées autrefois » (JACCOTTET, 2012: 87) comme si l'extensité temporelle déjà exprimée favorisait le retour en arrière au passé.

\section{2. Éléments de synthèse}

L'objectif du présent travail n'a pas été de recenser toutes les manifestations discursives de la douceur mais plutôt de montrer que celle-ci est d'abord un effet de discours qui, pour se réaliser, emprunte des voies variablement tensives. Celles-ci dépendent principalement de la corrélation entre l'intensité et l'extensité d'une part, et les subvalences qu'elles sous-tendent, à savoir la tonicité, le tempo, la temporalité et la spatialité, d'autre part. Grâce à ces catégories reconnaissables en discours, on a vu comment Jaccottet a pu sémiotiser l'expérience perceptive qu'implique la douceur, l'expérience consistant essentiellement en une action effectuée par le monde sensible sur la conscience du sujet par l'intermédiaire du corps propre (BARBARA, 2009 : 32) : la douceur implique nécessairement une relation à l'altérité (DUFOURMANTELLE, $2013: 29$ ). Le sujet percevant éprouve d'abord la douceur et la transmet ensuite en discours mais le monde qu'il perçoit n'est pas un monde dépourvu du sens mais plutôt un monde tensif, à savoir un monde nourri de tensions multiples que le sujet éprouve (ex. la tension entre la tonicité faible et forte, le tempo lent et rapide,...): le sens n'est pas une couche projetée sur la réalité mais se construit à même cette réalité au moyen de notre corps propre (BORDRON, 2007), le sens est donc atteint au sein du sensible (BARBARA, 2009: 31, 32). L'approche tensive que nous adoptons considère donc la douceur comme un devenir qui accepte des degrés, des gradations d'intensité et d'extensité qui se manifestent discursivement et que l'on ne pourrait comprendre sans prendre en considération l'expérience perceptive.

Dans le recueil, la douceur dépend, le plus souvent, d'une tonicité (lumineuse, auditive, ...) faible et d'un tempo lent mais cette intensité affaiblie cède la place à une extensité, surtout spatiale, élargie ou parfois diffuse. Parfois, l'intensité est remise en cause par cette sorte d'extensité cosmique: la douceur jaccottéenne s'avère ici plutôt extensive qu'intensive, embrassant tout le monde, ayant la capacité de «recoudre l'univers » (JACCOTTET, 2012 : 57) : la temporalité et la spatialité tendent à se généraliser. La concentration sur l'objet se dissout en extensité élargie, ce qui constitue le ressort le plus important de la douceur dans le recueil (MINANO, 1998 : 79) : le sujet percevant commence par une visée plus ou moins tonique de l'objet et se termine par une saisie atone (FONTANILLE, ZILBERBERG, 1998 : 96). L'entrée des grandeurs se fait souvent d'une manière progressive (ex : l'apparition du cerisier). Cette idée d'une intensité diffractée en extensité semble correspondre à l'hypothèse de Samson (2004) selon laquelle la pensée de Jaccottet, loin de 
reconnaître un seul centre organisateur, en reconnaît une multiplicité comme issue d'un éclatement (63).

Parfois, le tempo atteint un degré de lenteur qui frôle l'atonie, l'absence totale du mouvement (tempo $=0$ ), ce qui confirme en partie la remarque de Sourdillon (1996) concernant les images qui apparaissent soudain au poète et qui suspendent momentanément le cours du temps (36) : on l'a vu, par exemple, avec le verger du cognassier. Ce schéma illustre la corrélation inverse entre l'intensité, ici le tempo, et l'extensité, ici la durée : plus le tempo est rapide, moins la durée est longue. Selon Zilberberg (2011), le tempo lent rétrécit l'espace (29) mais ce qu'on a vu chez Jaccottet semble le contraire : le tempo lent est souvent lié à l'expansion spatiotemporelle.

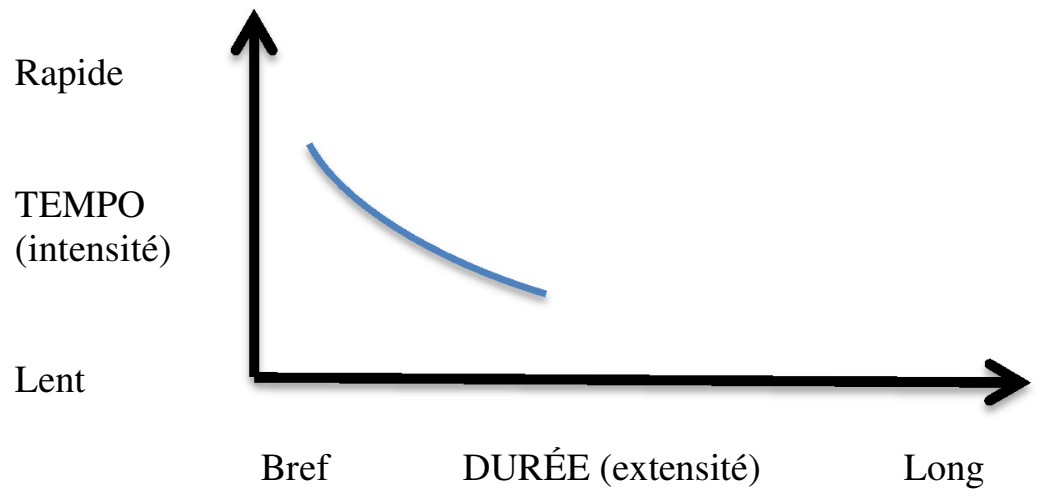

La douceur extensive dans le recueil semble emprunter le parcours suivant : accès, expansion, diffusion (FONTANILLE, ZILBERBERG, 1998: 113): les grandeurs sémiotiques accèdent dans l'espace tensif du sujet, s'étendent spatialement dans la plupart des cas puis se diffusent jusqu'à embrasser l'univers: la douceur jaccottéenne se place alors dans la région de la dispersion, définie essentiellement par la faiblesse d'une part et la diffusion d'autre part (ZILBERBERG, 2012 : 19) : le schéma suivant illustre la région de la douceur : à l'intersection de la faiblesse d'intensité et de la diffusion extensive, ce qui nous placent dans le cadre des valeurs d'univers qui dépendent de la corrélation converse entre le faible et le diffus et acceptent volontiers le mélange extensif (FONTANILLE, ZILBERBERG, 1998 : 33).

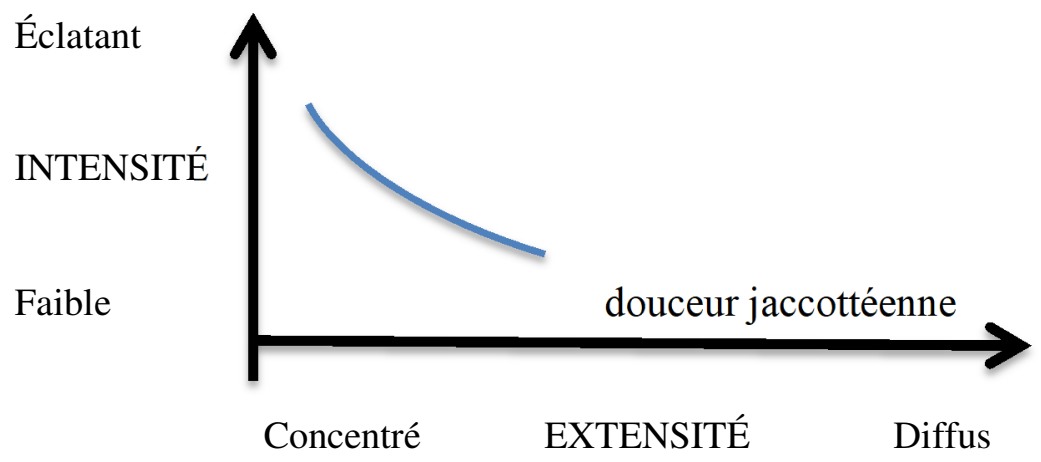


Dans le cadre de cette approche, la douceur est donc étudiée non en tant que catégorie figurative ou discursive mais plutôt figurale, c'est-à-dire, une catégorie qui se place à l'amont de l'énonciation : il y a lieu donc de considérer la douceur comme un langage profond et plus dense, un langage secret dont le poète s'attacherait à perfectionner l'emploi tout en restant attentif à l'expérience essentielle (JOSSUA, 2002:31). Au moyen de ce langage profond, le poète arrive à mener le lecteur à éprouver sensiblement la douceur. Au dire de Dufourmantelle, la douceur s'avère « une passivité active à force de tensions» (2013:15) : cette hypothèse s' applique mieux à la conception de la douceur présentée dans le recueil : un espace de tension entre force et étendue et un mouvement dynamique d'harmonie (DUFOURMANTELLE, 2013: 39): si cet équilibre n'existe pas, on tomberait immanquablement dans la vacuité : l'extensité se transformerait alors en une vague diffusion, si la douceur n'avait pas, comme point de départ, un minimum de tonicité et de tempo. On pourrait ainsi rapprocher la douceur de l'idée du suspens, fréquent dans la poésie jaccottéenne : il désigne l'avènement d'un équilibre fragile (LACAUX, 1986 : 50, 51 ; GUERMÈs, 2012 : 108 ; ex. : les expressions spatiales de l'entredeux). Par nature, la douceur est éphémère : elle s'avère la plus haute expression de la sensibilité, son intelligence et sa force et néanmoins elle peut à chaque moment disparaître (DUFOURMANTELLE, 2013 : 63). Cependant, nous avons vu qu'avec Jaccottet la douceur accède au rang de l'état : son départ est certes perceptif mais vite elle s'étend spatialement et temporellement...

\section{BIBLIOGRAPHIE}

BARBARA Renaud (2009), La perception. Essai sur le sensible, Paris, Vrin, coll. Philosophie.

BEIVIDAS Waldir (2012), Sémiotique du vécu : phénoménologie ou sémiologie?, Proceedings of the 10 th world congress of the International Association for Semiotic Studies (IASS/AIS), Universidade da Coruña, Espagne, p. 707-718.

BORDRON Jean-François (2007), Le statut sémiotique du monde naturel et la question de l'objet, publié en ligne le 22/ 10 / 2007, Nouveaux actes sémiotiques, 110, https : //www. unilim. fr/actes-semiotiques/1572, consulté le 19/4/ 2019.

ChALARD Reynald André (2005), Philippe Jaccottet, la transparence, l'image et l'amour de l'insaisissable, Études françaises, vol. 41, no 3 , Montréal, PU, p. $129-151$.

COUÉGNAS Nicolas (2008), L'impression textuelle des paysages: analyse sémiotique et sémantique de poésies contemporaines françaises, https : //www. unilim. fr/actes-semiotiques/3387, mis en ligne le 14/3/2008, consulté le 20/4/2019.

COUÉGNAS Nicolas, LAURENT François (2012), exercices de sémantique tensive, https : //halshs. archives-ouvertes. fr/halshs-00708466/document, mis en ligne le 15/ 6/2012, consulté le 20/ 11/ 2018.

DuBOIS Jean (1987), (sous la direction de), Dictionnaire du français contemporain, Paris, Larousse. 
Dufourmantelle Anne (2013), Puissance de la douceur, Paris, Payot\& Rivages, coll. Manuels.

FONTANILle Jacques (1999), Sémiotique du discours, Limoges, Pulim.

FONTANILlE Jacques, ZILBERBERG Claude (1998), Tension et signification, Paris, Mardaga, coll. Philosophie et langage.

GuERMES Sophie (2012), La poésie de Philippe Jaccottet : réparer l'absence, « à la frontière de Dieu », Quêtes littéraires, 2, 2012, p. 101-115.

JacCOTTET Philippe (2012), Cahier de Verdure, Paris, Gallimard, coll. Poésie.

JossuA Jean-Pierre (2002), Pour lire Philippe Jaccottet. Figures présentes, figures absentes, Paris, L'Harmattan, coll. Critiques littéraires.

LACAUX André (1986), Le regard et son éthique dans la poésie de Ph. Jaccottet, in : DuMAs Marie-Claire, (Études recueillies par), La poésie de Philippe Jaccottet, Paris, Honoré Champion, coll. Unichamp, p. 45-59.

LEMAIRE Jean-Pierre (2014), Philippe Jaccottet à l'écoute de la lumière cachée, Études, 4207, juillet-août, p. 73-81.

Minano Evelio (1998), L'expérience poétique du fragment dans Cahier de Verdure de Philippe Jaccottet, Queste, p. 77-87.

MONTE Michèle (1999), Chercher à saisir/effacer des traces. Prose et poésie dans Cahier de Verdure de Philippe Jaccottet, Babel [en ligne], $\mathrm{n}^{\circ} 3$, 1999, mis en ligne le 25 septembre 2012, consulté le 23/2/2018: http: //journals. openeditions. org/babael/1495, p. 71-90.

MouTAT Audrey (2011), La traduction du sensible à l'œuvre dans les poèmes de Jaccottet, Littérature, 163, septembre/3, p. 51-61.

SAMSON Hélène (2004), Le « tissu poétique » de Philippe Jaccottet, Paris, Mardaga, coll. Philosophie du langage.

SOURDILLON Jean-Marc (1996), Philippe Jaccottet, une écriture de l'événement « le passage », Littérature, 104, décembre 1996, p. 32-46.

VIART Dominique (2003), La parole effacée de Philippe Jaccottet, in: BlanCKEMANN Bruno, (sous la direction de), Lectures de Philippe Jaccottet, Rennes, PU, coll. Didact. Français, p. 23-38.

ZILBERBERG Claude (2007), Éléments de grammaire tensive, Limoges, Pulim, coll. NAS.

ZILBERBERG Claude (2008), Pour saluer l'événement, publié en ligne le 1/4/2008, consulté le 30 /11/2018, https : //www. unilim. fr/actes-semiotiques/1601

ZiLberberg Claude (2011), Des formes de vie aux valeurs, Paris, Puf, coll. Formes sémiotiques.

ZILBERBERG Claude (2012), La structure tensive, Liège, PU. 